\title{
ESCALA DE COMPORTAMENTOS SOCIALMENTE RESPONSÁVEIS DO CONSUMIDOR: ESTUDO PRELIMINAR DE EVIDÊNCIA DE VALIDADE ${ }^{1}$
}

\author{
Fabiana Queiroga* \\ Valdiney Veloso Gouveia" \\ Maria da Penha de Lima Coutinho" \\ Tatiana Cristina Vasconcelos ${ }^{\mathfrak{}}$ \\ Girlene Ribeiro de Jesus ${ }^{\mathscr{E}}$
}

\begin{abstract}
RESUMO. Este estudo teve como objetivo adaptar para o Brasil a Escala de Comportamentos Socialmente Responsáveis do Consumidor - ECSRC (Roberts, 1996), cujo objetivo principal é avaliar em que medida os consumidores tendentes a expressar condutas indicativas de preocupação de ordem social também as levam em consideração no momento de adquirir seus produtos. Participaram 298 estudantes universitários, com idade média de 21 anos (d.p. = 4,60), sendo a maioria do sexo feminino $(72,4 \%)$. Por meio de uma análise fatorial dos eixos principais (PAF) com rotação promax foram identificados três fatores que explicam conjuntamente $41,1 \%$ da variância total. O primeiro fator agrupou cinco itens e foi denominado como preocupação ambiental $(\alpha=0,90)$, o segundo, preocupação com reciclagem, ficou composto por quatro itens $(\alpha=0,75)$; e, por fim, o fator denominado de preocupação social $(\alpha=0,70)$ reuniu seis itens da escala. Estes resultados são coerentes com a concepção de que múltiplos fatores são necessários para avaliar o comportamento do consumidor, ademais sugerem a adequação da ECSRC ao contexto brasileiro.
\end{abstract}

Palavras-chave: Preocupação ambiental e social, reciclagem, validade de construto.

\section{SOCIALLY RESPONSIBLE CONSUMER BEHAVIOR SCALE: A PRELIMINARY STUDY FOR EVIDENCE OF VALIDITY}

\begin{abstract}
The objective of this study was to adapt The Socially Responsible Consumer Behavior Scale -ECSRC (Roberts, 1996) to Brazil. The main objective of this scale is to evaluate to what degree consumers who tend to express conduct which indicates social responsibility take this conduct into consideration at the moment of product acquisition. Two hundred ninety-eight university students (average 21 yrs old, d.p. $=4.60,72.4 \%$ female) participated in this study. Three factors, which explain $41.1 \%$ of the total variance were found through an analysis of the principal axes (PAF) with a Promax rotation. The first factor grouped five items together and was called environmental concern ( $\alpha=0.90)$, the second, concern about recycling, was composed of four items $(\alpha=0.75)$ and, finally, six items from the scale were grouped together in the third factor called social concern $(\alpha=0.70)$. These results are coherent with the conception that multiple factors are necessary to evaluate consumer behavior, and also, suggest the adequacy of the ECSRC to the Brazilian context.
\end{abstract}

Key words: environmental and social concern, recycling, constructs validation.

Nos últimos anos o interesse por fatores que determinam a escolha do consumidor por determinados produtos tem se consolidado, refletido tanto na quantidade quanto na qualidade

\footnotetext{
Apoio CNPq.

* Mestranda da Universidade de Brasília.

\# Doutor em Psicologia Social. Docente da Universidade Federal da Paraíba.

II Doutora em Psicologia. Docente da Universidade Federal da Paraíba.

æ Doutoranda da Universidade Federal do Rio Grande do Sul.

Æ Doutoranda da Universidade de Brasília.
} 
de pesquisas que vêm sendo levadas a curso. Nas primeiras publicações sobre o tema podia ser observada uma tendência a compreender o comportamento de compra fundamentado num único modelo ou numa "grande teoria", em que se procurava contemplar tópicos, como ocupações e serviços sociais, mas eram omitidas questões mais gerais, como escolha e persuasão, que seriam fenômenos intrinsecamente relacionados ao comportamento de consumir (Allen, 2000; Simpson, Carmon, Dhar, Drolet \& Nowlis, 2001). Assim, as variáveis e os processos englobados nos estudos guiados por este modelo único estariam basicamente voltados para a busca de uma melhor compreensão sobre formação e mudança de atitudes em relação à maneira como as escolhas são feitas (Chaiken, Wood \& Eagly, 1996; Simpson \& cols., 2001).

A partir dos anos 1980 a ênfase numa compreensão por meio de um único modelo começa a perder sua força e surgem novas concepções, advogando que o comportamento do consumidor é complexo demais para ser significativamente capturado por este tipo de abordagem, que em última instância é incapaz de abarcar a gama de elementos da psicologia humana envolvidos neste comportamento. Desta forma, intensificaram-se as pesquisas que procuravam dar conta desses elementos, que, ainda com o apoio de disciplinas correlatas, poderiam ser agrupados de acordo com os tópicos de maior ênfase. Numa revisão feita nos principais meios de publicação da área - Journal of Consumer Research (de 1974 a 1999), Journal of Marketing Research (de 1969 a 1999) e Journal of Consumer Psychology (de 1990 a 1999) -, pesquisadores deste tema puderam agrupar trabalhos sobre comportamento do consumidor de acordo com os domínios "social" versus "cognitivo" (ver Simpson \& cols, 2001). A propósito, concluíram que a proporção de publicações com foco em tópicos sociais (por exemplo, consumo relacionado à família e influência social, grupos de referência, atribuição) tem diminuído, ao passo que os tópicos cognitivos (por exemplo, pesquisas que incluem comportamento de tomada de decisão, memória e conhecimento, busca de variedades e ainda processos pré-conscientes) têm experimentado aumento significativo.

Conforme apontam Simpson e cols. (2001), uma das maiores mudanças na área foi a diminuição de pesquisas cujo foco sejam os tópicos de atitudes. Nos anos 1970 e início dos anos 1980, o modelo de atitude multiatributo e a teoria da ação racional de Fishbein e Ajzen (1975) receberam grande atenção por parte dos pesquisadores de comportamento do consumidor, que procuravam examinar se as intenções mediariam o efeito das atitudes no comportamento e qual o papel do componente normativo na formação de atitudes. Contudo, estes modelos começam a ser superados e o foco de atenção amplia-se para os processos de persuasão instigados através do marketing, e não utilizando apenas os modelos derivados das teorias psicológicas.

Simpson e cols. (2001) apontam ainda outro tipo de agrupamento através de aspectos de comportamento de consumo "frio" versus "quente". Os aspectos do tipo "frio" incluiriam tópicos como o papel das crenças na formação de atitudes, atenção, percepção, aquisição de informação, aprendizagem, atribuição e regras de decisões. Já os aspectos do tipo "quente" incluiriam o papel do afeto e do humor, da tristeza, aspectos hedônicos do consumo, conflitos e motivos auto-expressivos para preferências por marcas registradas. Utilizando-se este agrupamento para a classificação das pesquisas levantadas na revisão comentada anteriormente, observa-se uma crescente ênfase no papel das emoções e outros aspectos "quentes" da cognição.

De acordo com Roberts (1996), a maioria das pesquisas realizadas nos Estados Unidos mostra que os consumidores têm deixado cada vez mais suas preocupações sociais afetar suas decisões de compras. De acordo com este autor, durante a década de 1970 era grande a preocupação com a proteção ao meio ambiente por parte da população norte-americana. Contudo, com o número crescente de iniciativas legais implementadas em resposta às diversas manifestações para que fossem corrigidos os problemas, esta questão pareceu estar contornada durante alguns anos. $\mathrm{Na}$ década de 1990 - portanto, coincidindo com o aumento de pesquisas sobre comportamento do consumidor com ênfase no papel das emoções -, com a ocorrência de desastres ambientais com ampla difusão pela mídia (por exemplo, o aumento do buraco da camada de ozônio, os numerosos acidentes com espalhamento de petróleo no mar, os desastres ambientais causados por guerras sucessivas), o interesse público parece se voltar novamente para a questão da preocupação ambiental, pensada inclusive em termos mais inclusivos e amplos (ver Bamberg, 2003).

Destarte, a repercussão de eventos como estes incentivou um comportamento consumista mais 
consciente e instigando a preferência dos consumidores por produtos de empresas que demonstram responsabilidade social. Nesse sentido, as empresas de marketing dos Estados Unidos têm apostado que a preocupação social seria um guia na tomada de decisão dos consumidores, exigindo assim a definição e o delineamento de estratégias que visem atingir este novo perfil do consumidor (Roberts, 1996). Nestes termos, conhecer as características sociodemográficas dos consumidores passou a ser mais um tópico importante para os estudos sobre comportamento do consumidor, ou seja, procurar levantar que tipo de público é mais preocupado com responsabilidades sociais antes da aquisição dos seus produtos e quais consumidores não levam em conta esta questão.

No Brasil, especificamente no âmbito da Psicologia, ainda são escassas as publicações acerca de comportamentos do consumidor. Entretanto, considerando-se as dimensões ampliadas de uma economia e campanha publicitária global, parece cada dia mais emergente a necessidade de fortalecer o conhecimento nesta área. Um passo fundamental a respeito é contar com instrumentos psicometricamente adequados, o que motivou o presente estudo. Seu objetivo principal foi adaptar para o contexto brasileiro a Escala de Comportamentos Socialmente Responsáveis do Consumidor, elaborada originalmente por Roberts (1996). Este autor sugere que os itens desta medida podem ser reunidos em fatores, inclusive propõe que estes podem ser explicados por diferentes variáveis; entretanto não oferece nenhuma análise ou resultado que suporte sua estrutura fatorial.

\section{MÉTODO}

\section{Amostra}

Participaram deste estudo 298 estudantes de uma universidade pública do Estado da Paraíba, distribuídos eqüitativamente nas áreas de ciências humanas e da saúde (aproximadamente 50\% para ambas). A amostra não é probabilística, mas sim, de tipo intencional, da qual convenientemente participaram os estudantes que, presentes em sala de aula, concordaram voluntariamente em responder ao instrumento. A média de idade destes foi de 21 anos $(D P=4,60)$, sendo a maioria do sexo feminino $(72,4 \%)$.

\section{Instrumentos}

Os participantes responderam a um questionário constando de duas partes, como segue.

A primeira parte é a Escala de Comportamentos Socialmente Responsáveis do Consumidor - ECSRC. Elaborado por Roberts (1996). Este instrumento contém 18 sentenças que visam avaliar com que frequiência a pessoa se comporta de maneira social e ambientalmente consciente no momento de comprar seus produtos (por exemplo: "Faço o possível para comprar produtos feitos de papel reciclado"; "Quando posso escolher, dou preferência a produtos que causam menor quantidade de poluição"; "Não compro produtos que utilizam propaganda mostrando grupos marginalizados - como negros, mulheres e gays - de uma forma negativa"). Apesar de alguns itens iniciarem com denotação de negatividade ("Não compro produtos..."), quase todos os itens da escala estão medindo no mesmo sentido, ou seja, medem a preocupação com a responsabilidade social. A única exceção é o item "Compro produtos para a minha família que prejudiquem o meio ambiente", que procura identificar a falta de responsabilidade com o meio ambiente. Os itens são respondidos numa escala de freqüência contendo cinco pontos, cujos extremos correspondem a $\mathbf{1}=$ Nunca e $\mathbf{5}=$ Sempre. Conquanto o autor tenha aplicado previamente esta escala em uma amostra de 605 pessoas da população geral norte-americana, nenhuma informação foi encontrada sobre sua validade de construto e consistência interna.

A segunda parte se constitui das Características sociodemográficas. Uma folha contendo questões de caráter sociodemográfico (por exemplo, idade, sexo, renda e estado civil) foi apresentada no final do questionário.

\section{Procedimento}

Inicialmente foram apresentados sumariamente os objetivos do estudo aos responsáveis pelas salas de aula da Universidade. Com a anuência destes, os pesquisadores se apresentaram e solicitaram a colaboração dos estudantes no sentido de que preenchessem um questionário, identificado como destinado a conhecer como estes pensam e se sentem acerca de questões do seu dia-a-dia. Embora a participação tenha se dado em contexto coletivo de sala de aula, solicitou-se que as respostas fossem dadas individualmente. A participação foi voluntária e se asseguraram o anonimato e sigilo das respostas, que 
deveriam ser tratadas no conjunto. Durante a coleta de dados as intervenções feitas destinaram-se unicamente a prestar esclarecimentos de forma, e nunca de conteúdo, procurando evitar diferentes conotações ou significados que pudessem comprometer suas respostas. Em média, 15 minutos foram suficientes para concluir sua participação.

\section{RESULTADOS}

\section{Análises dos pressupostos}

Através da distribuição e das pontuações padronizadas das variáveis foi realizada a identificação dos outliers univariados. Contudo, não foram observados casos que indicassem a necessidade de retirada de respondentes, ou seja, variáveis padronizadas superiores a 3,29 ou inferiores a $-3,29$. Com relação ao tamanho da amostra, inicialmente foi adotado um mínimo de 300 pessoas para se efetuarem as análises fatoriais, como apontam Tabachnick e Fidell (2000). Não obstante, apesar de a coleta de dados ter sido finalizada com 298 sujeitos, isto não pode ser considerado uma limitação ao prosseguimento das análises; em realidade, um número igual ou superior a 200 participantes é recomendado para obter soluções estáveis em análises multivariadas, a exemplo da análise fatorial (Gorsuch, 1983; Watkins, 1989). Em relação aos dados faltosos, devido à baixa porcentagem desses casos na amostra (máximo de 2\%, no item 12 da ECSRC), optou-se por substituí-los, utilizando-se o método da média.

Finalmente, por meio dos histogramas das variáveis, bem como das medidas de curtose e assimetria, observou-se que nenhuma variável apresentou problemas sérios a esse respeito, não sendo adotada então qualquer transformação. Além disso, a Análise Fatorial é robusta em relação à fuga desse pressuposto, a não ser que haja valores muito altos de curtose e assimetria (Tabachnick \& Fidell, 2000).

\section{Análises principais}

A fatorabilidade da matriz foi verificada através de correlações entre as variáveis, do KMO e do Teste de Esfericidade de Bartlett. A matriz de correlações para a Escala de Comportamentos Socialmente Responsáveis do Consumidor mostrou que, conquanto algumas correlações não tenham atingido o tamanho de 0,30 , muitas delas (aproximadamente 25\%) superaram este valor, o que pode indicar a presença de fatorabilidade (Tabachnick \& Fidell, 2000; Pasquali, 2002). O KMO encontrado foi de 0,83 e o Teste de Esfericidade de Bartlett, que verifica a hipótese de que a matriz de covariâncias é uma matriz de identidade, teve sua hipótese rejeitada, $\chi^{2}(153)=$ $1879,12 p<0,001$. O valor encontrado para o determinante, 0,00154 , é adequado e indica ao menos que o posto da matriz é menor do que o número das variáveis que a compõem, ou seja, há presença de fatores.

Considerando os indicadores supracitados, decidiu-se realizar uma Análise Fatorial Exploratória (AFE). Optou-se pelo método principal axis factoring (PAF), tendo sido fixada a rotação promax, admitindo a possibilidade de correlações significativas entre os fatores da ECSRC. Na Figura 1 é apresentado o screeplot dos valores próprios (eigenvalues) observados.

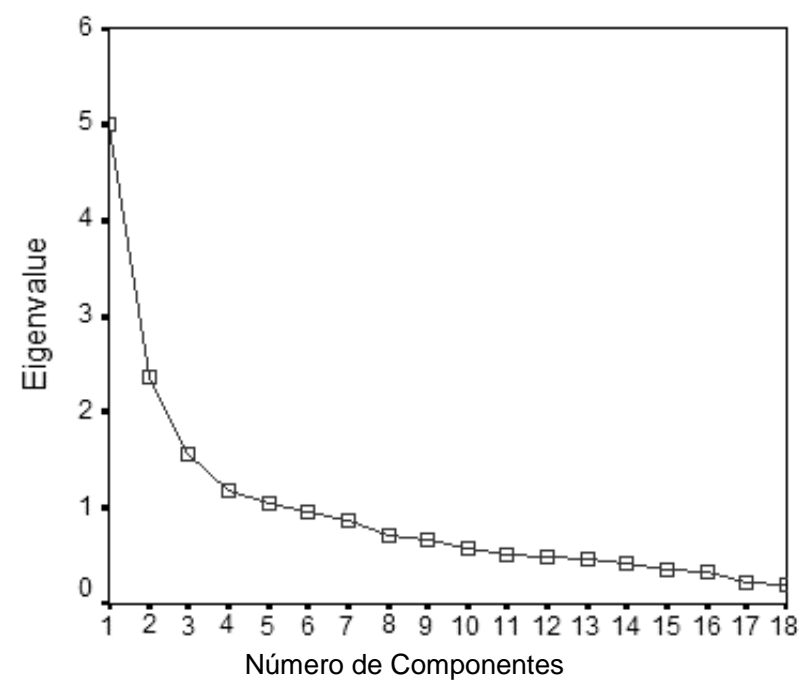

Figura 1. Representação Gráfica dos Eigenvalue

De acordo com a Figura 1, é possível observar que o gráfico aponta para uma solução entre dois e cinco fatores. Contudo, segundo o critério da variância total explicada, verificou-se que uma solução com mais do que três fatores não traria acréscimo substancial ao ganho final. Foi requerido, então, um valor mínimo da carga fatorial de 0,32 (indicando que pelo menos $10 \%$ de variância do item são compartilhados com o fator) para que o item fizesse parte do fator, conforme sugerem Tabachnick e Fidell (2000). Também foi estipulada a extração de três fatores. Os resultados a respeito podem ser observados na Tabela 1 a seguir. 
Tabela 1. Carga Fatorial (Matriz Pattern) e Comunalidade $\left(\mathrm{h}^{2}\right)$ dos Itens da ESCALA de Comportamento Social Responsável do Consumidor

\begin{tabular}{|c|c|c|c|c|}
\hline \multirow{2}{*}{ Descrição dos itens } & \multicolumn{3}{|c|}{ Fatores } & \multirow{2}{*}{$\mathbf{h}^{2}$} \\
\hline & $\mathbf{I}$ & II & III & \\
\hline $\begin{array}{l}\text { 02. Quando posso escolher, dou preferência a produtos que } \\
\text { causam menor quantidade de poluição. }\end{array}$ & $0,92^{*}$ & $-0,13$ & $-0,00$ & 0,86 \\
\hline $\begin{array}{l}\text { 04. Tento ser atencioso para comprar produtos que poluam } \\
\text { menos. }\end{array}$ & $0,86^{*}$ & 0,04 & $-0,01$ & 0,74 \\
\hline $\begin{array}{l}\text { 03. Quando posso escolher entre dois produtos semelhantes, } \\
\text { compro o que é menos prejudicial para as outras pessoas e } \\
\text { para o meio ambiente. }\end{array}$ & 0 & $-0,07$ & 0,01 & 0,66 \\
\hline $\begin{array}{l}\text { 01. Escolho meus produtos em função da pouca poluição que } \\
\text { eles causam. }\end{array}$ & $0,76^{*}$ & 0,03 & $-0,00$ & 0,58 \\
\hline $\begin{array}{l}\text { 05. Tenho escolhido determinados produtos por razões } \\
\text { ecológicas. }\end{array}$ & $0,66^{*}$ & 0,13 & 0,03 & 0,45 \\
\hline 11. Tento comprar produtos que podem ser reciclados. & $-0,02$ & $0,88 *$ & 0,02 & 0,78 \\
\hline $\begin{array}{l}\text { 09. Faço o possível para comprar produtos feitos de papel } \\
\text { reciclado. }\end{array}$ & 0,11 & $0,67 *$ & 0,04 & 0,46 \\
\hline $\begin{array}{l}\text { 08. Sempre que possível, compro produtos empacotados em } \\
\text { embalagens reutilizáveis. }\end{array}$ & $-0,09$ & $0,60^{*}$ & $-0,09$ & 0,38 \\
\hline 10. Utilizo algum meio de reciclagem para o meu lixo de casa. & 0,12 & $0,47 *$ & 0,08 & 0,24 \\
\hline $\begin{array}{l}\text { 16. Não compro produtos de companhias que não aceitam } \\
\text { funcionários negros, gays ou mulheres. }\end{array}$ & 0,01 & 0,00 & $\begin{array}{l}0,67 \\
*\end{array}$ & 0,45 \\
\hline $\begin{array}{l}\text { 15. Não compro produtos de companhias que utilizam mão de } \\
\text { obra infantil. }\end{array}$ & $-0,05$ & $-0,01$ & $\begin{array}{c}0,61 \\
*\end{array}$ & 0,37 \\
\hline $\begin{array}{l}\text { 14. Não compro produtos onde as propagandas mostram } \\
\text { mulheres de forma negativa. }\end{array}$ & 0,02 & $-0,05$ & $\begin{array}{l}0,56 \\
*\end{array}$ & 0,32 \\
\hline $\begin{array}{l}\text { 13. Não compro produtos que utilizam propaganda mostrando } \\
\text { grupos marginalizados (como negros, mulheres, gays) de } \\
\text { uma forma negativa. }\end{array}$ & 0,01 & 0,08 & $\begin{array}{c}0,54 \\
*\end{array}$ & 0,30 \\
\hline $\begin{array}{l}\text { 17. Não compro um produto quando fico sabendo que os } \\
\text { trabalhadores moram e trabalham em más condições. }\end{array}$ & $-0,02$ & 0,06 & $\begin{array}{c}0,44 \\
*\end{array}$ & 0,20 \\
\hline $\begin{array}{l}\text { 18. Não compro um produto quando sei que a companhia que } \\
\text { o vende é socialmente irresponsável. }\end{array}$ & $-0,03$ & $-0,13$ & $\begin{array}{l}0,38 \\
*\end{array}$ & 0,16 \\
\hline $\begin{array}{l}\text { 06. Se soubesse dos problemas que alguns produtos podem } \\
\text { causar para o meio ambiente eu não os compraria. }\end{array}$ & 0,31 & 0,06 & 0,01 & 0,10 \\
\hline $\begin{array}{l}\text { 12. Se possível não uso produtos que prejudicam outras } \\
\text { pessoas. }\end{array}$ & 0,26 & 0,26 & $-0,06$ & 0,14 \\
\hline $\begin{array}{l}\text { 07. Compro produtos para a minha família que prejudiquem o } \\
\text { meio ambiente. }\end{array}$ & $-0,23$ & $-0,07$ & 0,16 & 0,08 \\
\hline Número de itens & 5 & 4 & 6 & \\
\hline \multirow[t]{2}{*}{ Eigenvalue } & & 1,71 & & \\
\hline & 4,56 & & 1,11 & \\
\hline \% Variância total & 25,4 & 9,5 & 6,2 & \\
\hline \multirow[t]{2}{*}{ Alfa de Cronbach } & & 0,75 & & \\
\hline & 0,90 & & 0,70 & \\
\hline
\end{tabular}

Nota: * Item com carga fatorial considerada satisfatória e considerado para interpretar o fator.

Os três fatores solicitados explicaram conjuntamente $41,1 \%$ da variância total. Segundo a Tabela 1, os itens de número 6,7 e 12 não apresentaram cargas fatoriais consideradas satisfatórias - isto é, superiores a 0,329 - em ao menos um dos três fatores. Portanto, estes foram descartados. As maiores cargas fatoriais dos itens foram consideradas para identificar e nomear cada um dos fatores, conforme especificação a seguir:

- Fator I. Cinco itens compuseram este fator, que apresentou um eigenvalue de 4,56, sendo responsável pela explicação de 25,4\% da variância total. Expressa o cuidado com o meio ambiente por parte do consumidor ao comprar seus produtos (por exemplo: "Quando posso escolher, dou preferência a produtos que causam menor quantidade de poluição"; "Tenho escolhido determinados produtos por razões ecológicas"). Sua consistência interna, avaliada através do Alfa de Cronbach, foi de 0,90. A denominação deste fator parece clara: preocupação ambiental.

- Fator II. Compôs-se de quatro itens, com eigenvalue de 1,71, que corresponde à explicação de $9,5 \%$ da variância total. Expressa igualmente a preocupação do consumidor com o meio ambiente, porém com o foco na reciclagem de produtos (por exemplo: "Faço o possível para comprar produtos feitos de papel reciclado"; "Utilizo algum meio de reciclagem para o meu lixo de casa"). Apresentou Alfa de Cronbach de 0,75 e decidiu-se nomeá-lo como preocupação com reciclagem.

- Fator III. Este último fator está formado por seis itens, com eigenvalue de 1,11, tendo explicado $6,2 \%$ da variância total. Diz respeito à preocupação do consumidor com questões relacionadas ao bemestar social das pessoas envolvidas tanto na confecção do produto quanto na sua divulgação/publicidade (por exemplo: "Não compro produtos que utilizam propaganda mostrando grupos marginalizados - como negros, mulheres, gays - de uma forma negativa"; "Não compro um produto quando fico sabendo que os trabalhadores dessa empresa moram e trabalham em más condições). Observou-se um Alfa de Cronbach para este fator de 0,70. Decidiu-se denominá-lo como preocupação social.

\section{ANÁLISES COMPLEMENTARES}

É possível indagar acerca de uma dimensão geral, de ordem superior, que reúna os três fatores antes descritos. Esta possibilidade é descartada por meio das correlações que estes apresentaram entre si: a correlação mais forte $(r=0,45, p<0,001)$ foi entre os fatores I (preocupação ambiental) e II (preocupação com reciclagem). O Fator III (preocupação social) apresentou correlações inversas e, embora significativas, eram fracas, com os fatores I e II ( $r=$ $0,13, p=0,03$ para ambas). Em termos da importância destes fatores, observaram-se diferenças nas médias entre os três, Lambda de Wilks $=0,47, F(2,296)=$ 164, 94, p $<0,001$. Especificamente, a preocupação ambiental $(M=2,73 ; E P=0,06)$ foi mais 
preponderante do que a preocupação com reciclagem $(M=2,57 ; E P=0,05)$, que, por sua vez, importou mais que a preocupação social $(M=1,54 ; E P=0,03)$ no comportamento de compra e consumo (teste post hoc de Bonferroni; $p<0,05)$.

Finalmente, procurou-se conhecer em que medida as pontuações nos fatores de comportamento socialmente responsável do consumidor se correlacionariam com características demográficas dos participantes no estudo. Três variáveis principais foram consideradas: idade, renda declarada e sexo, esta última tratada como dummy. Em comum, o fato de todos os coeficientes de correlação terem sido fracos $\left(p_{\text {médio }}=0,07, p>0,05\right)$. A única correlação estatisticamente significativa foi observada para a idade e o fator preocupação ambiental $(r=0,12, p<$ $0,05)$, sugerindo que quanto mais velhas, mais as pessoas tendem a ter em conta questões ambientais no momento de decidir o que e onde comprar.

\section{DISCUSSÃO}

Segundo Roberts (1996), cada vez mais as decisões dos consumidores são afetadas por preocupações de ordem social, como, por exemplo, a preservação do meio ambiente. Portanto, torna-se relevante contar com instrumentos fidedignos que auxiliem na identificação do perfil de consumidores com tais preocupações. Nesse sentido, tendo-se em vista a escassez de literatura no Brasil a respeito de comportamentos do consumidor, objetivou-se com o presente estudo oferecer uma medida psicometricamente adequada que contribuísse para as pesquisas na área e que, alternativamente, suscitasse a discussão sobre o grau de preocupação com questões sociais que potencialmente podem influenciar os consumidores no momento de adquirirem seus produtos.

A Escala de Comportamentos Socialmente Responsáveis do Consumidor, embora contemple fatores distintos (preocupação ambiental, preocupação com reciclagem e preocupação social), parece avaliar com precisão um aspecto mais amplo subjacente ao ato de consumir que a literatura vem apontando (Bamberg, 2003; Roberts, 1996): a preocupação com as questões de ordem social como influenciador no momento da aquisição de produtos. A AFE revelou uma "estrutura simples" (Pasquali, 2002), com fatores nítidos, facilmente interpretados e teoricamente coerentes. Portanto, parece justificável afirmar que a ECSRC apresenta evidência de validade de construto. Seus fatores, embora curtos, formados por um número pequeno de itens, demonstram índices de consistência interna plenamente aceitáveis. Em suma, ao menos no contexto universitário paraibano, esta é uma medida psicometricamente adequada, sendo recomendável seu emprego em estudos futuros.

A preocupação ambiental parece mesmo ser um tema que preocupa as gerações atuais. A exemplo do que tem descrito Roberts (1996) acerca da preocupação dos norte-americanos com questões desta ordem, observou-se que também este foi o tema mais freqüentemente relacionado com os comportamentos de consumidor dos participantes do presente estudo. Pondere-se, contudo, que a preocupação ambiental, quer no plano das atitudes quer no dos comportamentos de consumidor auto-relatados, está apenas fracamente correlacionada com os comportamentos de compra em si (Roberts, 1996). Assim sendo, é importante não superestimar o dado de que os brasileiros têm em conta esta preocupação no momento de efetivarem uma compra; a respeito, três elementos clássicos do mercado seguem sendo preponderantes: preço, qualidade e conveniência.

Embora as pesquisas e as práticas na área de vendas e marketing sugiram a importância da segmentação do mercado em razão de variáveis demográficas dos potenciais consumidores, na pesquisa de Roberts (1996) estes atributos se revelaram pouco importantes para explicar os comportamentos socialmente responsáveis. Especificamente, este autor observou que de cinco variáveis antecedentes consideradas - sexo, idade, renda, ocupação e nível educacional - as duas últimas não contribuíram estatisticamente. As outras três explicaram conjuntamente $8 \%$ das respostas dos participantes. As mulheres e as pessoas mais velhas apresentaram comportamentos mais socialmente responsáveis, enquanto as pessoas com maior poder aquisitivo fizeram o contrário. $\mathrm{O}$ presente estudo revelou que apenas a idade contribuiu para explicar tais comportamentos, indicando coerentemente que as pessoas mais velhas expressaram mais preocupação ambiental. Este é um aspecto complementar desta pesquisa, que reforça também a adequação do instrumento de medida que está sendo apresentado.

\section{CONSIDERAÇÕES FINAIS}

A consideração da discussão sobre os resultados previamente descritos deve levar em conta eventuais limitações deste estudo. Um aspecto pode merecer maior atenção, a saber, a composição da amostra de participantes. Devido ao grau de instrução formal e poder aquisitivo apresentado, os estudantes universitários se configuram como um grupo de 
potenciais consumidores; são certamente mais conscientes do que a maior parte da população brasileira não-universitária a respeito das implicações sociais e do poder que pode ter sua decisão no momento de escolher produtos a comprar (por exemplo, demonstram uma orientação social mais horizontal, primando pela igualdade de condições entre as pessoas e condições de vida; ver Triandis, Chen \& Chan, 1998). Contudo, devido a ser um grupo minoritário no país, têm menor representatividade do consumidor brasileiro. Assim, considerar pessoas da população geral poderia ser oportuno e assegurar a adequação da ECSRC.

Vale ressaltar igualmente que as características sociodemográficas da amostra de participantes podem impor restrições aos achados deste estudo. As pesquisas realizadas previamente sobre o comportamento do consumidor tendem a levar em consideração o controle de variáveis como idade e renda, visto que compreendem características muito associadas com o poder de escolha do consumidor (Magnusson \& Hursti, 2002; Roberts, 1996). Na seleção da amostra de universitários não foi possível levar em conta estas variáveis. Uma amostragem mais criteriosa teria o conveniente de representatividade da população para a qual se pretende generalizar os resultados, porém seria mais laboriosa. Cabe destacar, contudo, que o propósito desta pesquisa não foi precisamente generalizar os resultados, mas conhecer a estrutura de comportamentos socialmente responsáveis do consumidor (Roberts, 1996), operacionalizados por meio de uma medida que se revelou multidimensional, composta por três fatores.

Não obstante as limitações apontadas anteriormente, é interessante reforçar que os índices psicométricos encontrados, a saber, a consistência interna dos fatores (com alfas de Cronbach iguais ou acima de 0,70), as cargas fatoriais observadas e ainda os demais índices verificados entre os fatores (Lambda de Wilks, teste post hoc de Bonferroni) ajudam a fundamentar a premissa de que a ECSRC apresenta uma estrutura consistente e confiável. Portanto, sugere-se que, se a estrutura final da ECSRC for considerada em estudos futuros, pode auxiliar outros pesquisadores na compreensão deste construto.

\section{REFERÊNCIAS}

Allen, M. W. (2000). The attribute-mediation and product meaning approaches to the influences of human values on consumer choices. In F. Columbus (Ed.), Advances in psychology research, (Vol. I, pp.31-76). Huntington: New Science Publishers.

Bamberg, S. (2003). How does environmental concern influence specific environmentally related behaviors? A new answer to an old question. Journal of Environmental Psychology, 23, 21-32.

Chaiken, S., Wood, W. \& Eagly, A. H. (1996). Principles of persuasion. In E. T. Higgins \& A. W. Kruglanski (Eds.), Social psychology: Handbook of basic principles (pp. 702-742). New York: Gilford.

Fishbein, M. \& Ajzen, I. (1975). Belief, attitude, intention and behavior: An introduction to theory and research. Reading, MA: Addison-Wesley.

Gorsuch, R. L. (1983). Factor analysis. Hillsdale, NJ: Lawrence Erlbaum Associates.

Magnusson, M. K. \& Hursti, U. K. K. (2002). Consumer attitudes towards genetically modified foods. Appetite, 39, 924.

Pasquali, L. (2002). Análise fatorial para pesquisadores. Brasília: LabPAM.

Roberts, J. A. (1996). Will the real socially responsible consumer please step forward? Business Horizons, 39, 79-83.

Simpson, I., Carmon, Z., Dhar, R., Drolet, A. \& Nowlis, S. M. (2001). Consumer research: In search of identity. Annual Review Psychology, 75, 52-249.

Tabachnick, B. G. \& Fidell, L.S. (2000). Using multivariate statistics $\left(4^{\text {rd }}\right.$ ed.). Northridge, CA: HarperCollins College.

Triandis, H. C., Chen, X. P. \& Chan, D. K. (1998). Scenarios for the measurement of individualism and collectivism. Journal of Cross-Cultural Psychology, 29, 275-289.

Watkins, D. (1989). The role of confirmatory factor analysis in cross-cultural research. International Journal of Psychology, 24, 685-701.

Recebido em 06/05/2004 Aceito em 30/10/2004

Endereço para correspondência: Fabiana Queiroga. SQN 410, Bloco E, Apto. 105, Asa Norte, CEP 70.865-050, Brasília-DF. E-mail: fabiana@cespe.unb.br 